\title{
Study on the Architecture about Equipment Support Information System based on Cloud Computing
}

\author{
HuiminZhang ${ }^{1}$,ZeyaZhang ${ }^{1, a}$, Jing Jing ${ }^{1}$, HairongHu ${ }^{1}$ \\ ${ }^{1}$ Department of Information Engineering, Academy of Armored Force Engineering, Beijing100072, \\ China; \\ amacromesopotamia@gmail.com
}

Keywords: SOA; SaaS; Cloud Computing; Equipment Support Information System

\begin{abstract}
Cloud computing is a new computing model of network services. As the inheritanceand development of SOA, it has become a hot issue in the internet field. Firstly, this article supposed the questions in the Equipment Support Information System Architecture based on SOA. Secondly, this paper analyzed the probability ofthe cooperation between SOA and Cloud Computing,and then designed the Cloud Computing Architecture based on SOA. Finally, this architecture was applied in the Equipment Support Information System.

We have developed a series of equipment support information system in the information construction. However, it has come up with the question of "Information Island"because of different needs, techniques and standards of platforms. To solve this problem without duplication of investment premise breakthrough between heterogeneous systems, and achieving integration and data sharing between the various of equipment support systems, we has developed the equipment support business information systems based on SOA [1]. It has efficiently solved the problem of "Information Island" and system integration. However, with the increasing of equipment types and number,the data center space is increasingly scarce. The way of centralized storage has threatened the data's security. The efficiency of traditional data query will be lower with the increasing amount of data.Deploying new applications will occupy the server's memory, which makes the system run slower. If you buy a supercomputer using traditional methods to solve the above problem will spend a lot of money. The cloud computing [2,3] which has the performances of highscalability, high availability, high reliability etc. has become the best choice to solve the above problem. So, the studying of cloud computing architecture based on SOA reflects its real significance.
\end{abstract}

\section{Introduction of Related Technical}

The Way of SOA Service Integration. The Service-Oriented Architecture [6]supports loose coupling and location transparency. This architecture uses service registration to achieve loose coupling between the service providers and the service users: the business system publishes the service interface to the service registration; and other business systems finds the service which is useful for them through the service registration. Figure 1 shows the way of SOA service integration.
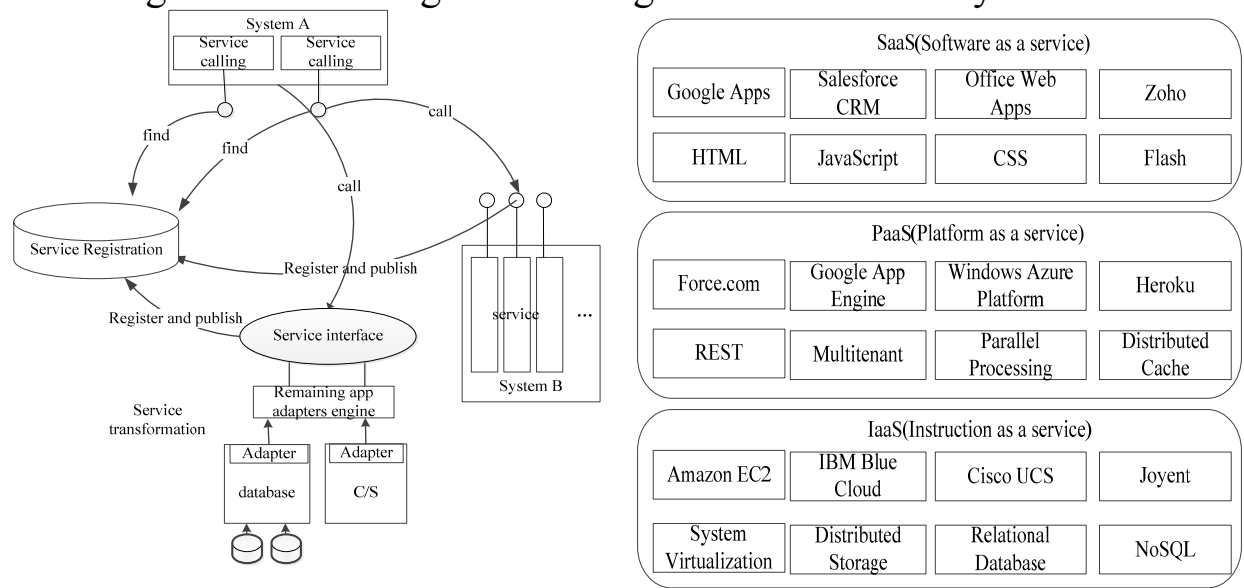

Fig. 1 The Way of SOA Service Integration

Fig. 2 Three Service Levels of Cloud Computing 
Service Levels of Cloud Computing. Cloud Computing integrates the technical advantages of Parallel Computing, DistributedComputing, Grid Computing, Utility Computing and soon.It is a kind of business modelcalled--"all resources are services"[4]. According to the type of service,Cloud Computingcan be divided into three different service levels[5]: SaaS(Software as a Service), PaaS (Platform as aService)and IaaS(Infrastructure as a Service). The three levels of Cloud Computing services as Figure 2:

a) SaaS(Software as a Service): The SaaS's service providers have deployed a variety of applications or systems in their own servers. The applications or systems aremanaged and maintainedby the service providers.In addition, the service providers also provide the hardware facilities which the applications need to run the system. What it provides is an application framework.

b) PaaS(Platform as a Service): The directcustomers of PaaS are system developers. The PaaS's providers provideusers with the required system development environment, hardware resources, server platforms, databases, application servers, and other services. The users can just simply develop the software systems on the platforms provided by the service providers. What it provides is an integrated environment.

c) IaaS (Infrastructure as a Service): The IaaS is commonly known as the hardware platform. It is mainly to provideusers with virtualized computing resources, communication resources, storage resources and network resources.Itincludesall the hardware facilities such as CPU, memories, servers, etc. What it provides is a software application environment.

\section{SOA and cloud computing Can be integrated}

The literature [9] has analyzed thefour different areas such as the cause, usage scenarios, key technologies, business models between SOA and cloud computing. They have different points, but there is still some possibility in the integration between them. It can be said that they can be integrated. The literature $[10,11,12]$ had explained the possibility and necessity between them, from business requirements, software development costs, quality of service (QoS) and other aspects.

Service is the core of SOA, which will encapsulate all resources as services. The enterprises make the different services which come from multiple services supporters as a service that is useful for them. In cloud computing, the service supporters published the resources as services. Many enterprises will buy the services such as hardware, platform, software and so on. Cloud computing supports lots of remote services which can be integrated SOA. As companies generally require the applications which are integrate by multiple services, cloud computing often provides a single service. So cloud computing cannot meet the requirements of the enterprise.However, SOA just be able to effectively integrate the services.

In addition, Web Service specification as one of the realization mechanisms of SOA has the features of standard, simple, cross-platform, cross-operating system, cross-language. It has been generally followed by grid computing and cloud computing. Therefore, cloud computing as a new Web Service architecture based on SOA, can easily coexist with other Web Service, and can be interoperated. For users, they just know what kind of service they need, just to see how to get services without having to know who provides the services in the end.

\section{Research of Cloud Computing Architecture based on SOA}

Use SOA to Integrate SaaS.Because Equipment Support Information System is a software system developed based on SOA, so how to set this system up in the clouds is the main problem to be solved in this article. The key to solving this problem lies in how to use SOA to integrate SaaS.

In the traditional sense,information integration is just system integration. However, SaaS is Web services which has been packaged.Its integration is a higher level of information integration. This level of integration can be used to solve by SOA. Using SOA to integrate SaaS service makes a large number of smaller size SaaS be integrated into one. So it can provide a more abstract, more granularities of Web services. SOA in Cloud Computing is different with SaaS: SOA is a model of 
software component; it changes the way to build internal systems, as well as the way how to interact with each other.However, SaaS is a model of software delivery, which emphasizes that software providers and users are separated and if the users use the service provided by providers they need to pay for it.

Although there are many differences between SOA and SaaS, for large-scale information systems, they have close relationship. Software systems can be serviced and then published by SaaS. It will providemany componentsfor SOA. SOA can find or use the services which have been published, and then combine them to produce new software which have more complete features. As a result, it significantly reduces the cost of software design and development. The new system can also be serviced and published.

As can be seen in Figure 3, the existing system can be transformed into appropriate standard Web services by the service tools of SaaS, and published to the service registry and provide consumers the services'description information which can be found. Service consumer can issue a request of service combination to build a new system. For example, the combination of service 1, service 2 and the service 3 shown in the figure. The process of finding service 1 is shown in dashed lines of no1 and no2. It shows that, before binding the service it firstly needs to find it from the service registry;other services have the same process. The new built systems can also be posted to the service registry and provided to consumers after being serviced by SaaS. It can be seen that,using SOA to integrate SaaS can quickly integrate the current services to build new generation of software systems which will meet the service consumers'needs. Figure 4 showsthe process ofthe integration between SOA and SaaS.
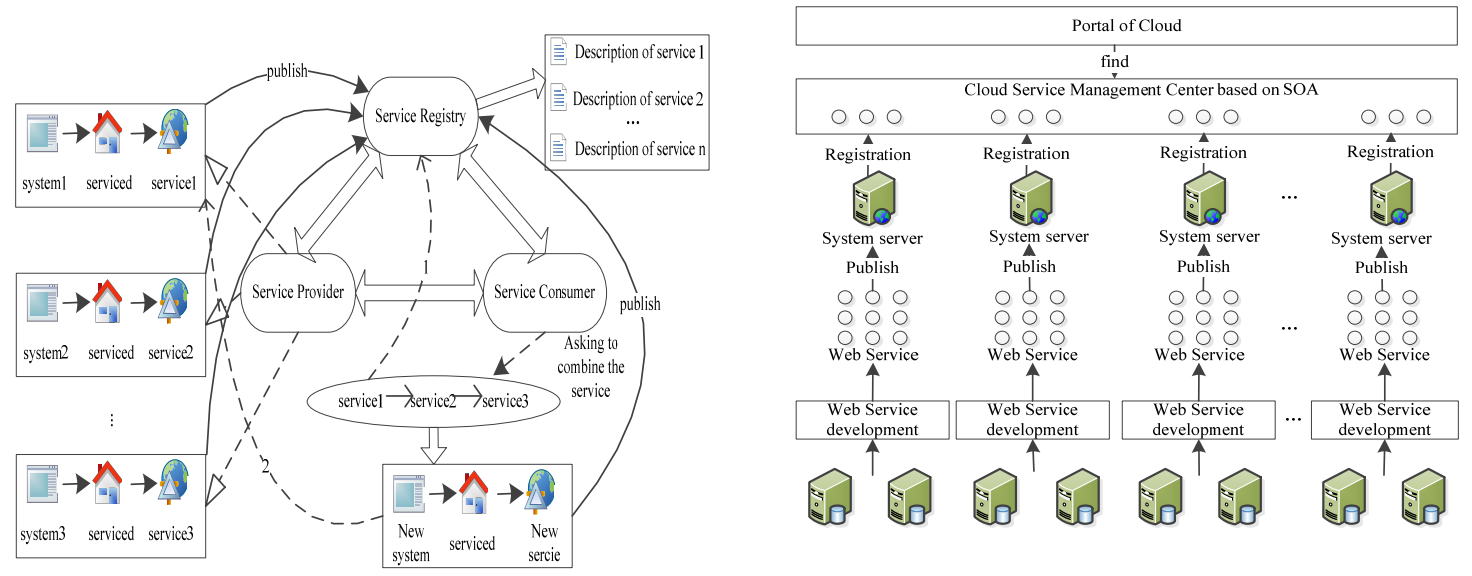

Fig. 3 SOA Integrates SaaS

Fig. 4 Process of SOA Combinesthe Software

Design of Cloud Computing Architecture based on SOA. From the above we have known that SOA can integrate the services provided by cloud computing, and there is property of integration between them. According to the characteristic that SOA and cloud computing can be integrated, the cloud computing architecture based on SOA when we design will include multiple levels of software applications and hardware systems.An available architecture we can reference shown in Figure 5. Bottom-up can be divided into: Hardware Platform layer, Cloud Management, Application Service layer, Service Scheduling layer, Service Interface layer and SOA Construct layer. 


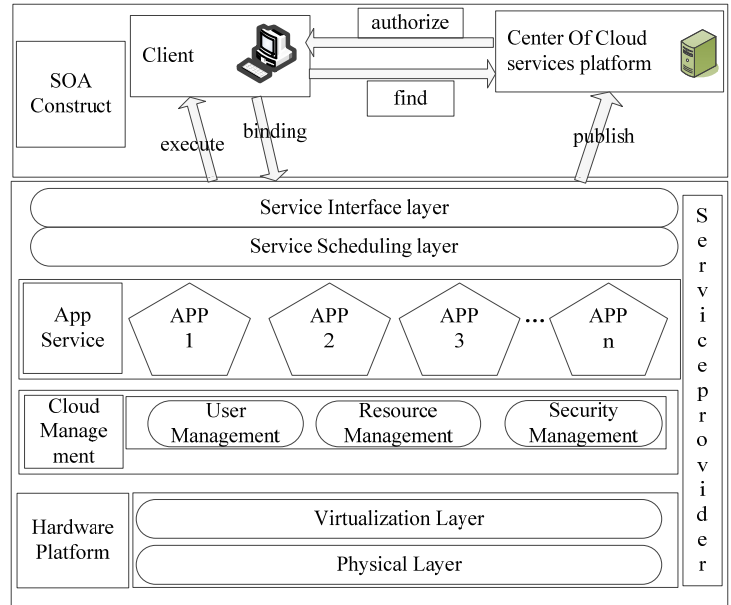

Fig. 5 Model of Cloud Computing Architecture based on SOA

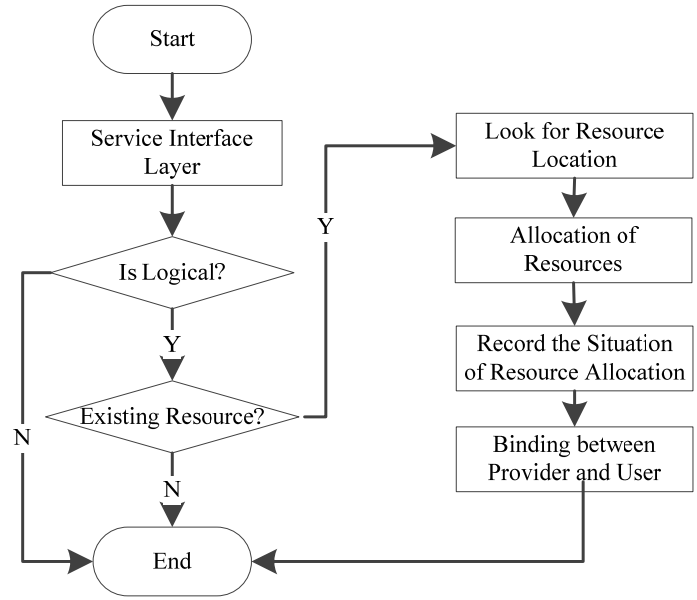

Fig. 6 Process of Resource Scheduling

(1) Hardware Platform Layer: that is, the so-called IaaSin the Cloud Computing,it includes physical and virtual service layer. It is the basic layer in the structure of Cloud Computing. Its main role is providing clients with virtualized storage resources, computing resources, network resources, and communicationresources. And the hardware it providescontainingstorage servers, CPU, computers,and so on. The CPU resources can provide the services ofdata computing and processing for the users, thecapacity and speed of data processing is much better than the client's computer. The memory resources can provide for usersfar more storage capacities than the client's. Computer cluster can provide distributed computing, greatly improving the speed of computing.

(2) Cloud Management Layer: that is, the so-called PaaSin the Cloud Computing. The function of this layer is that: it has the capability ofUser Management which containsAccount Management, User Interaction Management; CloudResource Management which contains Resource Deployment, Performance Optimization; Cloud Security Management which contains Identity Authentication, AccessAuthorityManagement, and Cloud Data Management which contains Server Deployment, Database Dispatcher Management. This layer ensures that the various resources can be applied safely and efficiently.

(3) Application Service Layer: that is, the so-called SaaS in the cloud. This layer provides for users services which are packaged by the SOA Structure Layer. The end-consumers can customize or get the services they need through a specific entrance into the Centerof Cloud Computing. Through the Cloud Computing application service layer, end-customers can save a lot of overhead used in the application development and improving machine performance to use the much better machine performance in the area of complex computing or storage work.

(4) Service Scheduling Layer: This layer manages and schedules the service resource, and accepts services submitted by service interface layer and determines its legitimacy.If it is legal, then this layer detects the current cloud resource pool whether there are resources needed for this request, if has been allocated the request is denied.If there are resources, then this layer will find its location 
and call the services for consumers. The specific process of scheduling is shown in Figure 6 .

(5) Service Interface Layer: This layer provides users with a standard, unified service interface; it is the channel of calling service. Clientsenter into the cloud computing service center to customize and consume required services through a dedicatedchannel.

(6) SOA Construct layer: This layer willregister service provided by provider to the center of cloud computing platformusing WSDLprotocol and then publish them using UDDI protocol.When consumers use the services, they can also find them using WSDL protocol. After finding the service, consumers can use SOAP protocol to interact with the service providerand use the service.

\section{Design of Equipment Support Information System Model based onCloud Architecture}

In this paper, we design the model of equipment support information system cloud architecture by combining the equipment support information system architecture and cloud computing architecture based on SOA. It is shown in Figure 7.

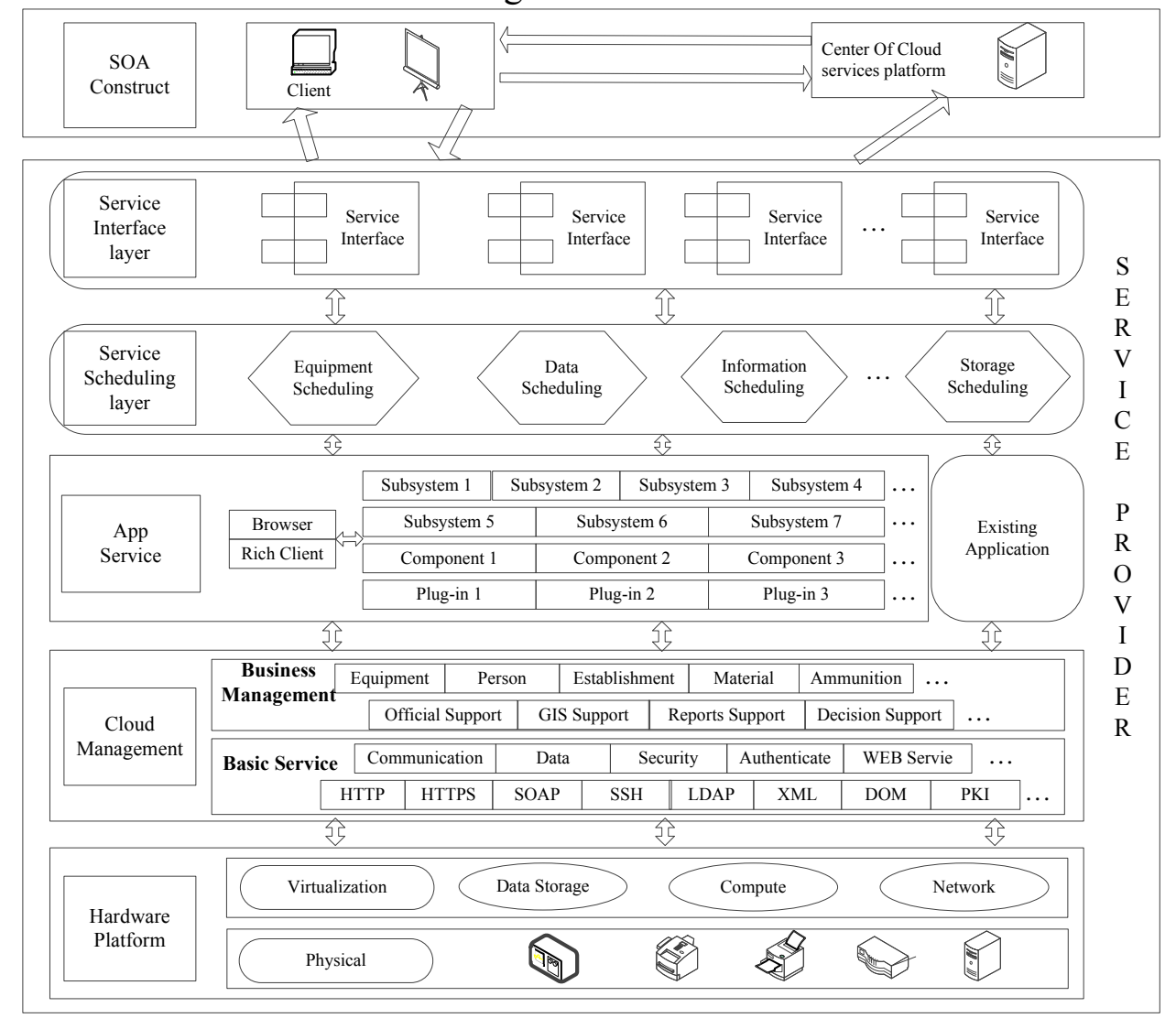

Fig. 7 Equipment Support Information System Model based on Cloud Architecture

General Headquarters, Military region, army, division and brigade etc. are the cloud service users. They look for the service they needed from the center of cloud service registry platform on the military network using computer, PDA and other devices. After finding the service, we can bind to the service interface layer. The center of Cloud services registry platform can be managed by one high-performance computer. Users can find the location of the service through the appropriate protocol and service description, and then select the service interface. The service interface layer can be managed by one node server computer. After accepting the request from the clients, the service interface layer forwards the request to the service scheduling layer.The service scheduling layer will verify the user's identity information. If the user's information is legitimate, the service scheduling layer will find the appropriate service.If the service does not exist or is being occupied, the service scheduling layer will response the fail message to the users.Otherwise, this layer will provide service to the users. This layer can be managed by multiple distributed computers. Application service layer and cloud management layer provide appropriate services to users by the scheduling algorithm. Theyare also managed by multiple distributed computer server. It includes 
person management, equipment management, material management, ammunition management, communication services, security services and other services. The hardware platform layer uses the core technology of cloud computing - virtualization technology to virtualize the CPU, memory, servers and other hardware to provide powerful capacity of computing and storage.

\section{Conclusion}

Cloud Computing is the core of a new generation of IT.As a new and promising service pattern, the Cloud Computing is the inheritance and development of SOAin some ways. The appearanceof Cloud Computingmust be able to promote the change of the computer applications. This paper analyzes the possibility of integration between SOAand Cloud Computingand then presents a design model of Cloud Computing architecture based on SOA.The conceptof the design model adoptsthe feature of loosecouplingof SOA.It increasesthe flexibility of the applications by packaging all types of resources as services, and publishesservicesthrough the "cloud". The integration of SOA and Cloud Computing will bring a bright future.However, we shouldn't be blindly optimistic, because the cloud is not very mature.And so far, there hasn't existed a uniform standard about Cloud Computing architecture,and the appearance of itincreases the difficulty of designsomewhat, so it requires a long process toseamlessly integrate SOA and Cloud Computing.

\section{References}

[1]Yang Xiaolong. Study on Service-Oriented General Equipment Support Information System Infrastructure [D] Beijing: Academy of Armored Forces Engineering .2012:55-59

[2]Amazon Elastic Compute Cloud [EB / OL] http://aws.amazon Com / ec2 /

[3] Google App Engine [EB / OL]. Http://appengine.google.com/

[4] Armbrust M, Fox A, Griffith R. A view of Cloud Computing[J]. Commune. ACM (CACM), $2010.53(4): 40-47$

[5] CUI Yunfei, LI Yi, etc.Cloud ComputingArchitecture Research Basedon Service Oriented Architecture [J]. Journal of the Academy of Equipment Command \& Technology, 2011 (4):77-80

[6]Lvxi Yan, Zhang Runtong.Integration of SOA-based Enterprise Information Resources [J] China Science and Technology Forum .2006.3 (104): 36-40

[7] Cui Yunfei, Li Yi and other studies of cloud computing architecture based on SOA [J] Equipment Command \& Technology .2011 (4): 77-80

[8]RobisonS. The Next Wave: Everything as a Service [EB/OL] http://www.hp.com/hpinfo/execteam/articles/robison/08eaas.html.

[9]ZHU Zhiliang, YUAN Haitao, etc. SOA and Cloud Computing:Competition or Integration [J]. Computer Science, 2011.12 (12):6-11

[10]Feuerlicht G. Next Generation SOA: Can SOA Survive Cloud Computing? [J]. Advances in Intelligent Web Mastering-2, AISC 67:19-29

[11] Li Wu-bin, Svard P. REST-based SOA Application in the Cloud: A Text Correction Service Case Study[C]//Proceedings of the 2010 6th World Congress on Service. Miami, Florida, USA, 2010:84-90

[12] Cao Bu-qing, Li Bing, Xia Qi-ming, A Service-oriented Qos-as-sured and Multi-agent Cloud Computing Architecture [J]. Cloud-Com, 2009:644-649 